\title{
Stability result of the Lamé system with a delay term in the internal fractional feedback
}

\author{
Abbes Benaissa \\ Laboratory of Analysis \\ and Control of PDEs, \\ Djillali Liabes University, \\ P. O. Box 89, Sidi Bel Abbes 22000, \\ Algeria \\ email: benaissa_abbes@yahoo.com
}

\author{
Soumia Gaouar \\ Laboratory of Analysis \\ and Control of PDEs, \\ Djillali Liabes University, \\ P. O. Box 89, Sidi Bel Abbes 22000, \\ Algeria \\ email: gaouar.s@gmail.com
}

\begin{abstract}
In this article, we consider a Lamé system with a delay term in the internal fractional feedback. We show the existence and uniqueness of solutions by means of the semigroup theory under a certain condition between the weight of the delay term in the fractional feedback and the weight of the term without delay. Furthermore, we show the exponential stability by the classical theorem of Gearhart, Huang and Pruss.
\end{abstract}

\section{Introduction}

In this article, we consider the initial boundary value problem for the Lamé system given by:

$$
\begin{cases}u_{t t}-\mu \Delta u-(\mu+\lambda) \nabla(\operatorname{div} u) & \\ +a_{1} \partial_{t}^{\sigma, k} u(x, t-\tau)+a_{2} u_{t}(x, t)=0 & \text { in } \Omega \times(0,+\infty) \\ u=0 & \text { in } \Gamma \times(0,+\infty) \\ u(x, 0)=u_{0}(x), \quad u_{t}(x, 0)=u_{1}(x) & \text { in } \Omega \\ u_{t}(x, t-\tau)=f_{0}(x, t-\tau) & \text { in } \Omega \times(0, \tau)\end{cases}
$$

2010 Mathematics Subject Classification: 35B40, 47D03, 74D05

Key words and phrases: Lamé system, fractional delay term, uniform stability, semigroup theory 
where $\mu, \lambda$ are Lamé constants, $u=\left(u_{1}, u_{2}, \ldots, u_{n}\right)^{\top}$. Here $\Omega$ is a bounded domain in $\mathbb{R}^{n}$ with smooth boundary $\Gamma=\partial \Omega$. Moreover, $a_{1}>0, a_{2}>0$ and the constant $\tau>0$ is the time delay. The notation $\partial_{t}^{\sigma, k}$ stands for the exponential fractional derivative operator of order $\sigma$. It is defined by

$$
\partial_{\mathrm{t}}^{\sigma, \kappa} w(\mathrm{t})=\frac{1}{\Gamma(1-\sigma)} \int_{0}^{\mathrm{t}}(\mathrm{t}-\mathrm{s})^{-\sigma} e^{-\kappa(\mathrm{t}-\mathrm{s})} \frac{\mathrm{d} w}{\mathrm{~d} s}(\mathrm{~s}) \mathrm{d} s \quad 0<\sigma<1, \quad \kappa>0 .
$$

Delay effects arise in many applications and pratical problems because, in most instances, physical, chemical, biological, thermal, and economic phenomena naturally depend not only on the present state but also on some past occurrences. In recent years, the control of PDEs with time delay effects has become an active area of research, see for example [1], [17], and references therein. In many cases it was shown that delay is a source of instability and even an arbitrarily small delay may destabilize a system which is uniformly asymptotically stable in the absence of delay unless additional conditions or control terms have been used.The stability issue of systems with delay is, therefore, of theoretical and practical importance. In particular, consider the wave equation with homogeneous Dirichlet boundary condition

$$
\begin{cases}u^{\prime \prime}(x, t)-\Delta_{x} u(x, t)+\mu_{1} u^{\prime}(x, t) & \\ +\mu_{2} u^{\prime}(x, t-\tau)=0 & \text { in } \Omega \times(0,+\infty), \\ u(x, t)=0 & \text { on } \Gamma \times(0,+\infty), \\ u(x, 0)=u_{0}(x), u^{\prime}(x, 0)=u_{1}(x) & \text { in } \Omega, \\ u^{\prime}(x, t-\tau)=f_{0}(x, t-\tau) & \text { in } \Omega \times(0, \tau) .\end{cases}
$$

For instance in [13] the authors studied the problem (PW). They determined suitable relations between $\mu_{1}$ and $\mu_{2}$, for which the stability or alternatively instability takes place. More precisely, they showed that the energy is exponentially stable if $\mu_{2}<\mu_{1}$ and they also found a sequence of delays for which the corresponding solution of (PW) will be instable if $\mu_{2}>\mu_{1}$. The main approach used in [13] is an observability inequality obtained with a Carleman estimate.

Noting that the case of the wave equation with internal fractional feedback (without delay) have treated in [8] where it is proven global existence and uniqueness results. As far as we are concerned, this is the first work in the literature that takes into account the uniform decay rates for Lamé system with delay term in the internal fractional feedback.

The remainder of the paper falls into five sections. In Section $\mathbf{2}$, we show that the above system can be replaced by an augmented one obtained by coupling an equation with a suitable diffusion, and we study of energy functional 
associated to system. In section 3, we state a well-posedness result for problem (P). In section $\mathbf{4}$, we prove the strong asymptotic stability of solutions. In section $\mathbf{5}$ we show the exponential stability using the Gearhart-Huang-Pruss theorem.

\section{Preliminary}

This section is concerned with the reformulation of the model (P) into an augmented system. For that, we need the following claims.

Theorem 1 (see [12]) Let $\omega$ be the function:

$$
\omega(\xi)=|\xi|^{(2 \sigma-1) / 2}, \quad-\infty<\xi<+\infty, 0<\sigma<1 .
$$

Then the relationship between the 'input' $U$ and the 'output' $O$ of the system

$$
\begin{gathered}
\partial_{\mathrm{t}} \psi(\xi, \mathrm{t})+\left(\xi^{2}+\mathrm{k}\right) \psi(\xi, \mathrm{t})-\mathrm{U}(\mathrm{t}) \omega(\xi)=0, \quad-\infty<\xi<+\infty, \kappa>0, \mathrm{t}>0, \\
\psi(\xi, 0)=0 \\
\mathrm{O}(\mathrm{t})=(\pi)^{-1} \sin (\sigma \pi) \int_{-\infty}^{+\infty} \omega(\xi) \psi(\xi, \mathrm{t}) \mathrm{d} \xi
\end{gathered}
$$

is given by

$$
\mathrm{O}=\mathrm{I}^{1-\sigma, \kappa} \mathrm{U}=\mathrm{D}^{\sigma, \kappa} \mathrm{U},
$$

where

$$
\left[I^{\sigma, \kappa} f\right](t)=\frac{1}{\Gamma(\sigma)} \int_{0}^{t}(t-s)^{\sigma-1} e^{-\kappa(t-s)} f(s) d s .
$$

Proof. From (2) and (3), we have

$$
\psi(\xi, t)=\int_{0}^{t} \omega(\xi) e^{-\left(\xi^{2}+\kappa\right)(t-s)} U(s) d s .
$$

Hence, by using (4), we get

$$
\mathrm{O}(\mathrm{t})=(\pi)^{-1} \sin (\sigma \pi) e^{-\kappa \mathrm{t}} \int_{0}^{\mathrm{t}}\left[2 \int_{0}^{+\infty}|\xi|^{2 \sigma-1} e^{-\xi^{2}(\mathrm{t}-\mathrm{s})} \mathrm{d} \xi\right] e^{\kappa s} \mathrm{u}(\mathrm{s}) \mathrm{ds}
$$


Thus,

$$
\begin{aligned}
\mathrm{O}(\mathrm{t}) & =(\pi)^{-1} \sin (\sigma \pi) e^{-\kappa t} \int_{0}^{t}\left[(t-s)^{-\sigma} \Gamma(\sigma)\right] e^{k s} \mathrm{U}(\mathrm{s}) \mathrm{ds} \\
& =(\pi)^{-1} \sin (\sigma \pi) \int_{0}^{t}\left[(\mathrm{t}-\mathrm{s})^{-\sigma} \Gamma(\sigma)\right] e^{-\kappa(t-s)} \mathrm{U}(\mathrm{s}) \mathrm{ds}
\end{aligned}
$$

which completes the proof. Indeed, we know that $(\pi)^{-1} \sin (\sigma \pi)=\frac{1}{\Gamma(\sigma) \Gamma(1-\sigma)}$.

Lemma 1 (see [5]) If $\left.\left.\lambda \in \mathrm{D}_{\mathrm{K}}=\mathbb{C} \backslash\right]-\infty,-\mathrm{k}\right]$ then

$$
\int_{-\infty}^{+\infty} \frac{\omega^{2}(\xi)}{\lambda+\kappa+\xi^{2}} d \xi=\frac{\pi}{\sin \sigma \pi}(\lambda+\kappa)^{\sigma-1} .
$$

We make the following hypotheses on the damping and the delay functions:

$$
\mathrm{a}_{1} \mathrm{k}^{\sigma-1}<\mathrm{a}_{2}
$$

We are now in a position to reformulate system (P). As in [13], we introduce the new variable

$$
z(x, \rho, t)=u_{t}(x, t-\rho \tau), \quad x \in \Omega, \rho \in(0,1), t>0 .
$$

Then the above variable $z$ satisfies

$$
\tau z_{\mathfrak{t}}(x, \rho, t)+z_{\rho}(x, \rho, t)=0, \quad x \in \Omega, \rho \in(0,1), t>0 .
$$

Consequently, by using Theorem 1 , the system ( $\mathrm{P}$ ) is equivalent to

$$
\begin{cases}u_{t t}-\mu \Delta u-(\mu+\lambda) \nabla(\operatorname{div} u) & \\ +\zeta \int_{-\infty}^{+\infty} \omega(\xi) \psi(x, \xi, t) d \xi+a_{2} u_{t}(t)=0 & \text { in } \Omega \times(0,+\infty), \\ \psi_{t}(x, \xi, t)+\left(\xi^{2}+k\right) \psi(x, \xi, t) & \\ -z(x, 1, t) \omega(\xi)=0 & \text { in } \Omega \times(-\infty, \infty) \times(0) \\ \tau z_{t}(x, \rho, t)+z_{\rho}(x, \rho, t)=0 & \text { in } \Omega \times(0,1) \times(0, \\ u(x, t)=0 l & \text { on } \Gamma \times(0,+\infty), \\ z(x, 0, t)=u_{\mathfrak{t}}(x, t), & \text { in } \Omega \times(0,+\infty), \\ u(x, 0)=u_{0}(x), \quad u_{t}(x, 0)=u_{1}(x) & \text { on } \Omega, \\ \psi(x, \xi, 0)=0 & \text { on } \Omega \times(-\infty, \infty), \\ z(x, \rho, 0)=f_{0}(x,-\rho \tau) & \text { in } \Omega \times(0,1),\end{cases}
$$


where $\zeta=(\pi)^{-1} \sin (\sigma \pi) a_{1}$.

We define the energy of the solution by:

$$
\begin{array}{r}
E(t)=\frac{1}{2} \sum_{j=1}^{n}\left(\left\|u_{j t}\right\|_{L^{2}(\Omega)}^{2}+\mu\left\|\nabla u_{j}\right\|_{L^{2}(\Omega)}^{2}+\zeta \int_{\Omega} \int_{-\infty}^{+\infty}\left|\psi_{j}(x, \xi, t)\right|^{2} d \xi d x\right) \\
+\frac{v}{2} \sum_{j=1}^{n} \int_{\Omega} \int_{0}^{1}\left|z_{j}(x, \rho, t)\right|^{2} d \rho d x+\frac{(\mu+\lambda)}{2}\|\operatorname{div} u\|_{L^{2}(\Omega)}^{2} .
\end{array}
$$

where $v$ is a positive constant verifying

$$
\tau \zeta\left(\int_{-\infty}^{+\infty} \frac{\omega^{2}(\xi)}{\xi^{2}+\kappa} d \xi\right)<v<\tau\left(2 a_{2}-\zeta\left(\int_{-\infty}^{+\infty} \frac{\omega^{2}(\xi)}{\xi^{2}+\kappa} d \xi\right)\right) .
$$

Remark 1 Using Lemma 1, the condition (11) means that

$$
\tau \kappa^{\sigma-1}<v<\tau\left(2 a_{2}-a_{1} \kappa^{\sigma-1}\right) .
$$

Lemma 2 Let $(u, \psi, z)$ be a regular solution of the problem $\left(\mathrm{P}^{\prime}\right)$. Then there exists a positive constant $\mathrm{C}$ such that the energy functional defined by (10) satisfies

$$
E^{\prime}(t) \leq-C \sum_{j=1}^{n} \int_{\Omega}\left(u_{t}^{2}+z(x, 1, t)^{2}\right) d x .
$$

Proof. Multiplying the first equation in $(\mathrm{P})$ by $\overline{\mathrm{u}}_{\mathrm{jt}}$, integrating over $\Omega$ and using integration by parts, we get

$$
\begin{aligned}
& \frac{1}{2} \frac{\mathrm{d}}{\mathrm{dt}}\left\|u_{j t}\right\|_{2}^{2}-\mu \Re \int_{\Omega} \Delta u_{j} \bar{u}_{j \mathrm{t}} \mathrm{d} x-(\mu+\lambda) \Re \int_{\Omega} \frac{\partial}{\partial x_{j}}(\operatorname{div} u) \bar{u}_{j \mathrm{t}} \mathrm{d} x \\
& \quad+\zeta \int_{\Omega} \bar{u}_{j \mathrm{t}} \int_{-\infty}^{+\infty} \omega(\xi) \psi_{j}(x, \xi, t) d \xi d x+a_{2} \int_{\Omega}\left|u_{j t}(t)\right|^{2} d x=0 .
\end{aligned}
$$

Then

$$
\begin{aligned}
& \frac{1}{2} \frac{d}{d t} \sum_{j=1}^{n}\left(\left\|u_{j t}\right\|_{L^{2}(\Omega)}^{2}+\mu\left\|\nabla u_{j}\right\|_{L^{2}(\Omega)}^{2}\right)+\frac{(\mu+\lambda)}{2}\|\operatorname{div} u\|_{L^{2}(\Omega)}^{2} \\
& \quad+a_{2} \sum_{j=1}^{n}\left\|u_{j t}\right\|_{L^{2}(\Omega)}^{2}+\zeta \Re \sum_{j=1}^{n} \int_{\Omega} \bar{u}_{j t} \int_{-\infty}^{+\infty} \omega(\xi) \psi_{j}(x, \xi, t) d \xi d x=0 .
\end{aligned}
$$


Multiplying the second equation in $\left(\mathrm{P}^{\prime}\right)$ by $\zeta \bar{\psi}_{j}$ and integrating over $\Omega \times$ $(-\infty,+\infty)$, we obtain:

$$
\begin{array}{r}
\frac{\zeta}{2} \frac{\mathrm{d}}{\mathrm{dt}} \sum_{j=1}^{\mathrm{n}}\left\|\psi_{j}\right\|_{\mathrm{L}^{2}(\Omega \times(-\infty,+\infty))}^{2}+\zeta \sum_{j=1}^{\mathrm{n}} \int_{\Omega} \int_{-\infty}^{+\infty}\left(\xi^{2}+\kappa\right)\left|\psi_{j}(x, \xi, t)\right|^{2} \mathrm{~d} \xi \mathrm{d} x \\
-\zeta \Re \sum_{j=1}^{n} \int_{\Omega} z_{j}(x, 1, \mathrm{t}) \int_{-\infty}^{+\infty} \omega(\xi) \bar{\psi}_{j}(x, \xi, t) d \xi \mathrm{d} x=0 .
\end{array}
$$

Multiplying the third equation in $\left(\mathrm{P}^{\prime}\right)$ by $v \bar{z}_{j}$ and integrating over $\Omega \times(0,1)$, we get:

$$
\frac{1}{2} \frac{\mathrm{d}}{\mathrm{dt}} \sum_{j=1}^{\mathrm{n}}\left\|z_{j}\right\|_{L^{2}(\Omega \times(0,1))}^{2}+\frac{\tau^{-1}}{2} \sum_{j=1}^{n} \int_{\Omega}\left(z_{j}^{2}(x, 1, t)-u_{j \mathrm{t}}^{2}(x, t)\right)=0 .
$$

From (10), (13) and (15) we get

$$
\begin{aligned}
& \mathcal{E}^{\prime}(t)=-a_{2} \sum_{j=1}^{n}\left\|u_{j t}\right\|_{L^{2}}^{2}-\zeta \sum_{j=1}^{n} \int_{\Omega} \int_{-\infty}^{+\infty}\left(\xi^{2}+\kappa\right)\left|\psi_{j}(x, \xi, t)\right|^{2} d \xi d x \\
& -\zeta \mathfrak{R} \sum_{j=1}^{n} \int_{\Omega} \bar{u}_{j t} \int_{-\infty}^{+\infty} \omega(\xi) \psi_{j}(x, \xi, t) d \xi d x \\
& +\zeta \Re \sum_{j=1}^{n} \int_{\Omega} z_{j}(x, 1, t) \int_{-\infty}^{+\infty} \omega(\xi) \bar{\psi}_{j}(x, \xi, t) d \xi d x \\
& +\frac{v \tau^{-1}}{2} \sum_{j=1}^{n} \int_{\Omega} u_{t}^{2}(x, t) d x-\frac{v \tau^{-1}}{2} \sum_{j=1}^{n} \int_{\Omega} z_{j}^{2}(x, 1, t) d x .
\end{aligned}
$$

Moreover, we have

$$
\left|\int_{-\infty}^{+\infty} \omega(\xi) \psi_{j}(x, \xi, t) d \xi\right| \leq\left(\int_{-\infty}^{+\infty} \frac{\omega^{2}(\xi)}{\xi^{2}+k} d \xi\right)^{\frac{1}{2}}\left(\int_{-\infty}^{+\infty}\left(\xi^{2}+k\right)\left|\psi_{j}(x, \xi, t)\right|^{2} d \xi\right)^{\frac{1}{2}} .
$$

Then

$$
\begin{aligned}
& \left|\int_{\Omega} z_{j}(x, 1, t) \int_{-\infty}^{+\infty} \omega(\xi) \bar{\psi}_{j}(x, \xi, t) d \xi d x\right| \\
& \leq\left(\int_{-\infty}^{+\infty} \frac{\omega^{2}(\xi)}{\xi^{2}+\kappa} d \xi\right)^{\frac{1}{2}}\left\|z_{j}(x, 1, t)\right\|_{L^{2}(\Omega)}\left(\int_{\Omega} \int_{-\infty}^{+\infty}\left(\xi^{2}+\kappa\right)\left|\psi_{j}(x, \xi, t)\right|^{2} d x d \xi\right)^{\frac{1}{2}}
\end{aligned}
$$


and

$$
\begin{aligned}
& \left|\int_{\Omega} \bar{u}_{j t}(x, t) \int_{-\infty}^{+\infty} \omega(\xi) \psi_{j}(x, \xi, t) d \xi d x\right| \\
& \leq\left(\int_{-\infty}^{+\infty} \frac{\omega^{2}(\xi)}{\xi^{2}+\kappa} d \xi\right)^{\frac{1}{2}}\left\|u_{j t}(x, t)\right\|_{L^{2}(\Omega)}\left(\int_{\Omega} \int_{-\infty}^{+\infty}\left(\xi^{2}+\kappa\right)\left|\psi_{j}(x, \xi, t)\right|^{2} d x d \xi\right)^{\frac{1}{2}} .
\end{aligned}
$$

Applying the Cauchy-Schwarz inequality we obtain

$$
\begin{aligned}
E^{\prime}(t) \leq & \left(-a_{2}+\frac{\zeta I}{2}+\frac{v \tau^{-1}}{2}\right) \sum_{j=1}^{n} \int_{\Omega} u_{j t}^{2}(x, t) d x \\
& +\left(\frac{\zeta I}{2}-\frac{v \tau^{-1}}{2}\right) \sum_{j=1}^{n} \int_{\Omega} z_{j}^{2}(x, 1, t) d x,
\end{aligned}
$$

where $I=\int_{-\infty}^{+\infty} \frac{\omega^{2}(\xi)}{\xi^{2}+\kappa} d \xi$, which implies

$$
E^{\prime}(t) \leq-C \sum_{j=1}^{n} \int_{\Omega}\left(u_{j t}^{2}(x, t)+z_{j}^{2}(x, 1, t)\right) d x
$$

with

$$
\mathrm{C}=\min \left\{\left(\mathrm{a}_{2}-\frac{\zeta \mathrm{I}}{2}-\frac{v \tau^{-1}}{2}\right),\left(-\frac{\zeta \mathrm{I}}{2}+\frac{\nu \tau^{-1}}{2}\right)\right\} .
$$

Since $v$ is chosen satisfying assumption (11), the constant $C$ is positive. This completes the proof of the lemma.

\section{Well-posedness}

In this section, we give the existence and uniqueness result for system $\left(\mathrm{P}^{\prime}\right)$ using the semigroup theory. Let us denote $\mathrm{U}=(u, v, \psi, z)^{\mathrm{T}}$, where $v=u_{\mathrm{t}}$. The system $\left(\mathrm{P}^{\prime}\right)$ can be rewrite as follows:

$$
\left\{\begin{array}{l}
\mathrm{u}^{\prime}=\mathcal{A} \mathrm{u}, \quad \mathrm{t}>0 \\
\mathrm{u}(0)=\left(\mathrm{u}_{0}, \mathrm{u}_{1}, \psi_{0}, \mathrm{f}_{0}\right)
\end{array}\right.
$$

where $\mathcal{A}: \mathrm{D}(\mathcal{A}) \subset \mathcal{H} \rightarrow \mathcal{H}$ is the linear operator defined by

$$
\mathcal{A}\left(\begin{array}{c}
u \\
v \\
\psi \\
z
\end{array}\right)=\left(\begin{array}{c}
v \\
\mu \Delta u+(\mu+\lambda) \nabla(\operatorname{div} u)-\zeta \int_{-\infty}^{+\infty} \omega(\xi) \psi(x, \xi) d \xi-a_{2} v \\
-\left(\xi^{2}+\kappa\right) \psi+z(x, 1) \omega(\xi) \\
-\tau^{-1} z_{\rho}(x, \rho)
\end{array}\right)
$$


and $\mathcal{H}$ is the energy space given by

$$
\mathcal{H}=\left(\mathrm{H}_{0}^{1}(\Omega)\right)^{\mathrm{n}} \times\left(\mathrm{L}^{2}(\Omega)\right)^{\mathrm{n}} \times\left(\mathrm{L}^{2}(\Omega \times(-\infty,+\infty))\right)^{\mathrm{n}} \times\left(\mathrm{L}^{2}(\Omega \times(0,1))\right)^{\mathrm{n}} .
$$

For any $\mathrm{u}=(u, v, \Psi, z)^{\top} \in \mathcal{H}, \tilde{\mathrm{u}}=(\tilde{\mathrm{u}}, \tilde{v}, \tilde{\psi}, \tilde{z})^{\top} \in \mathcal{H}$, we equip $\mathcal{H}$ with the inner product defined by

$$
\begin{aligned}
& <\mathrm{u}, \tilde{\mathrm{u}}>_{\mathcal{H}}=\sum_{j=1}^{n} \int_{\Omega}\left(v_{j} \overline{\tilde{v}}_{j}+\mu \nabla u_{j} \nabla \overline{\tilde{u}}_{j}\right) d x+(\mu+\lambda) \int_{\Omega}(\operatorname{div} u)(\operatorname{div} \overline{\tilde{u}}) d x \\
& +\zeta \sum_{j=1}^{n} \int_{\Omega} \int_{-\infty}^{+\infty} \psi_{j}(x, \xi) \overline{\tilde{\psi}}_{j}(x, \xi) d \xi d x+\zeta \sum_{j=1}^{n} \int_{\Omega} \int_{0}^{1} z(x, \rho) \overline{\tilde{z}}_{j}(x, \rho) d \rho d x .
\end{aligned}
$$

The domain of $\mathcal{A}$ is given by

$$
\mathrm{D}(\mathcal{A})=\left\{\begin{array}{l}
(u, v, \psi, z)^{\mathrm{T}} \text { in } \mathcal{H}: u \in\left(\mathrm{H}^{2}(\Omega) \cap \mathrm{H}_{0}^{1}(\Omega)\right)^{\mathrm{n}}, v \in\left(\mathrm{H}^{1}(\Omega)\right)^{\mathrm{n}}, \\
-\left(\xi^{2}+\kappa\right) \psi+z(x, 1, \mathrm{t}) \omega(\xi) \in\left(\mathrm{L}^{2}(\Omega \times(-\infty,+\infty))\right)^{\mathrm{n}}, \\
z \in\left(\mathrm{L}^{2}\left(\Omega ; \mathrm{H}^{1}(0,1)\right)\right)^{\mathrm{n}}, \\
|\xi| \psi \in\left(\mathrm{L}^{2}(\Omega \times(-\infty,+\infty))\right)^{\mathrm{n}}, v=z(., 0) \text { in } \Omega
\end{array}\right\} .
$$

We show that the operator $\mathcal{A}$ generates a $\mathrm{C}_{0}$ semigroup in $\mathcal{H}$. We prove that $\mathcal{A}$ is a maximal dissipative operator. For this we need the following two Lemmas.

Lemma 3 The operator $\mathcal{A}$ is dissipative and satisfies for any $\mathrm{U} \in \mathrm{D}(\mathcal{A})$,

$$
\mathfrak{R}\langle\mathcal{A} \mathrm{U}, \mathrm{U}\rangle_{\mathcal{H}} \leq-\mathrm{C} \sum_{j=1}^{n} \int_{\Omega}\left(v^{2}+z(x, 1)^{2}\right) \mathrm{d} x .
$$

Proof. For any $\mathrm{U}=(\mathrm{u}, \boldsymbol{v}, \psi, z) \in \mathrm{D}(\mathcal{A})$, using (17), (12) and the fact that

$$
\mathrm{E}(\mathrm{t})=\frac{1}{2}\|\mathrm{U}\|_{\mathcal{H}}^{2},
$$

estimate (20) easily follows.

Lemma 4 The operator $(\tilde{\lambda} \mathrm{I}-\mathcal{A})$ is surjective for $\tilde{\lambda}>0$.

Proof. For any $G=\left(G_{1}, G_{2}, G_{3}, G_{4}\right)^{\top} \in \mathcal{H}$, where $G_{i}=\left(g_{i}^{1}, g_{i}^{2}, \ldots, g_{i}^{n}\right)^{\top}$, we show that there exists $\mathrm{U} \in \mathrm{D}(\mathcal{A})$ satisfying

$$
(\tilde{\lambda} \mathrm{I}-\mathcal{A}) \mathrm{U}=\mathrm{G} .
$$


Equation (22) is equivalent to

$$
\left\{\begin{array}{l}
\tilde{\lambda} u-v=G_{1}(x) \\
\tilde{\lambda} v-\mu \Delta u-(\mu+\lambda) \nabla(\operatorname{div} u)+\zeta \int_{-\infty}^{+\infty} \omega(\xi) \psi(x, \xi) d \xi+a_{2} v=G_{2}(x) \\
\tilde{\lambda} \psi+\left(\xi^{2}+\kappa\right) \psi-z(x, 1) \omega(\xi)=G_{3}(x, \xi) \\
\tilde{\lambda} z(x, \rho)+\tau^{-1} z_{\rho}(x, \rho)=G_{4}(x, \rho)
\end{array}\right.
$$

Suppose $\boldsymbol{u}$ is found with the appropriate regularity. Then, $(23)_{1}$ and $(23)_{3}$ yield

$$
v=\tilde{\lambda} u-G_{1}(x) \in\left(H^{1}(\Omega)\right)^{n}
$$

and

$$
\psi=\frac{\mathrm{G}_{3}(x, \xi)+\omega(\xi) z(x, 1)}{\xi^{2}+\kappa+\tilde{\lambda}} .
$$

We note that the last equation in (23) with $z(x, 0)=v(x)$ has a unique solution given by

$$
z(x, \rho)=v(x) e^{-\tilde{\lambda} \rho \tau}+\tau e^{-\tilde{\lambda} \rho \tau} \int_{0}^{\rho} G_{4}(x, r) e^{\tilde{\lambda} r \tau} d r .
$$

Inserting (24) in (26), we get

$$
z(x, \rho)=\tilde{\lambda} u(x) e^{-\tilde{\lambda} \rho \tau}-G_{1}(x) e^{-\tilde{\lambda} \rho \tau}+\tau e^{-\tilde{\lambda} \rho \tau} \int_{0}^{\rho} G_{4}(x, r) e^{\tilde{\lambda} r \tau} d r, \quad x \in \Omega, \rho \in(0,1) .
$$

In particular,

$$
z(x, 1)=\tilde{\lambda} u(x) e^{-\tilde{\lambda} \tau}+z_{0}(x), \quad x \in \Omega
$$

with $z_{0} \in \mathrm{L}^{2}(\Omega)$ defined by

$$
z_{0}(x)=-G_{1}(x) e^{-\tilde{\lambda} \tau}+\tau e^{-\tilde{\lambda} \tau} \int_{0}^{1} G_{4}(x, r) e^{\tilde{\lambda} r \tau} d r, \quad x \in \Omega
$$

Inserting (24) in $(23)_{2}$, we get

$$
\begin{aligned}
& \left(\tilde{\lambda}^{2}+\tilde{\lambda} a_{2}\right) u-\mu \Delta u-(\mu+\lambda) \nabla(\operatorname{div} u)+\gamma a_{1} \int_{-\infty}^{+\infty} \omega(\xi) \psi(x, \xi) d \xi \\
& =G_{2}(x)+\left(\tilde{\lambda}+a_{2}\right) G_{1}(x) .
\end{aligned}
$$


Solving system (30) is equivalent to finding $u \in\left(H^{2} \cap H_{0}^{1}(\Omega)\right)^{n}$ such that

$$
\begin{aligned}
& \sum_{j=1}^{n} \int_{\Omega}\left(\left(\tilde{\lambda}^{2}+\tilde{\lambda} a_{2}\right) u_{j} \bar{w}_{j}-\mu \Delta u_{j} \bar{w}_{j}\right) d x-(\mu+\lambda) \int_{\Omega} \frac{\partial}{\partial x_{j}}(\operatorname{div} u) \bar{w}_{j} d x \\
& \quad+\zeta \sum_{j=1}^{n} \int_{\Omega} \bar{w}_{j} \int_{-\infty}^{+\infty} \omega(\xi) \psi_{j}(x, \xi) d \xi d x=\sum_{j=1}^{n} \int_{\Omega}\left(g_{2}^{j}(x)+\left(\tilde{\lambda}+a_{2}\right) g_{1}^{j}(x)\right) \bar{w}_{j} d x
\end{aligned}
$$

for all $w \in\left(H_{0}^{1}(\Omega)\right)^{n}$. Inserting (25) in (31) and using (28), we obtain that

$$
\left\{\begin{array}{l}
\sum_{j=1}^{n} \int_{\Omega}\left(\left(\tilde{\lambda}^{2}+\tilde{\lambda} a_{2}\right) u_{j} \bar{w}_{j}+\mu \nabla u_{j} \nabla \bar{w}_{j} d x\right)+(\mu+\lambda) \int_{\Omega}(\operatorname{div} u)(\operatorname{div} \bar{w}) d x \\
+\tilde{\lambda} \theta \sum_{j=1}^{n} \int_{\Omega} u_{j} \bar{w}_{j} e^{-\tilde{\lambda} \tau} d x=\sum_{j=1}^{n} \int_{\Omega}\left(g_{2}^{j}(x)+\left(\tilde{\lambda}+a_{2}\right) g_{1}^{j}(x)\right) \bar{w}_{j} d x \\
-\zeta \sum_{j=1}^{n} \int_{\Omega} \bar{w}_{j}\left(\int_{-\infty}^{\infty} \frac{\omega(\xi) g_{3}^{j}(x, \xi)}{\xi^{2}+\kappa+\tilde{\lambda}} d \xi\right) d x-\theta \sum_{j=1}^{n} \int_{\Omega} \bar{w}_{j} z_{0}(x) d x .
\end{array}\right.
$$

where $\theta=\zeta \int_{-\infty}^{+\infty} \frac{\omega^{2}(\xi)}{\xi^{2}+\kappa+\tilde{\lambda}} d \xi$. Problem (32) is of the form

$$
\mathcal{B}(u, w)=\mathcal{L}(w),
$$

where $\mathcal{B}:\left(H_{0}^{1}(\Omega)\right)^{n} \times\left(H_{0}^{1}(\Omega)\right)^{n} \rightarrow \mathbb{C}$ is the sesquilinear form defined by

$$
\begin{aligned}
\mathcal{B}(u, w) & =\sum_{j=1}^{n} \int_{\Omega}\left(\left(\tilde{\lambda}^{2}+\tilde{\lambda} a_{2}\right) u_{j} \bar{w}_{j}+\mu \nabla u_{j} \nabla \bar{w}_{j} d x\right) \\
& +(\mu+\lambda) \int_{\Omega}(\operatorname{div} u)(\operatorname{div} \bar{w}) d x+\tilde{\lambda} \theta \sum_{j=1}^{n} \int_{\Omega} u_{j} \bar{w}_{j} e^{-\tilde{\lambda} \tau} d x
\end{aligned}
$$

and $\mathcal{L}:\left(\mathrm{H}_{0}^{1}(\Omega)\right)^{\mathrm{n}} \rightarrow \mathbb{C}$ is the antilinear functional given by

$$
\begin{aligned}
\mathcal{L}(w) & =\sum_{j=1}^{n} \int_{\Omega}\left(g_{2}^{j}(x)+\left(\tilde{\lambda}+a_{2}\right) g_{1}^{j}(x)\right) \bar{w}_{j} d x \\
& -\zeta \sum_{j=1}^{n} \int_{\Omega} \bar{w}_{j}\left(\int_{-\infty}^{\infty} \frac{\omega(\xi) g_{3}^{j}(x, \xi)}{\xi^{2}+\kappa+\tilde{\lambda}} d \xi\right) d x-\theta \sum_{j=1}^{n} \int_{\Omega} \bar{w}_{j} z_{0}(x) d x .
\end{aligned}
$$


It is easy to verify that $\mathcal{B}$ is continuous and coercive, and $\mathcal{L}$ is continuous. Consequently, by the Lax-Milgram theorem, we conclude that for all $w \in\left(H_{0}^{1}(\Omega)\right)^{n}$, the system (33) has a unique solution $u \in\left(H_{0}^{1}(\Omega)\right)^{n}$. By the regularity theory for the linear elliptic equations, it follows that $u \in\left(H^{2}(\Omega)\right)^{n}$. Therefore, the operator $(\tilde{\lambda} I-\mathcal{A})$ is surjective for any $\tilde{\lambda}>0$. Consequently, using Hille-Yosida theorem, we have the following existence result:

\section{Theorem 2 (Existence and uniqueness)}

(1) If $\mathrm{U}_{0} \in \mathrm{D}(\mathcal{A})$, then system (17) has a unique strong solution

$$
\mathrm{U} \in \mathrm{C}^{0}\left(\mathbb{R}_{+}, \mathrm{D}(\mathcal{A})\right) \cap \mathrm{C}^{1}\left(\mathbb{R}_{+}, \mathcal{H}\right) .
$$

(2) If $\mathrm{U}_{0} \in \mathcal{H}$, then system (17) has a unique weak solution

$$
\mathrm{u} \in \mathrm{C}^{0}\left(\mathbb{R}_{+}, \mathcal{H}\right) .
$$

\section{Strong stability}

One simple way to prove the strong stability of (17) is to use the following theorem due to Arendt-Batty and Lyubich-Vũ (see [2] and [10]).

Theorem 3 ([2]-[10]) Let $\mathrm{X}$ be a reflexive Banach space and $(\mathrm{T}(\mathrm{t}))_{\mathrm{t} \geq 0}$ be a $\mathrm{C}_{0}$-semigroup generated by $\mathrm{A}$ on $\mathrm{X}$. Assume that $(\mathrm{T}(\mathrm{t}))_{\mathrm{t} \geq 0}$ is bounded and that no eigenvalues of $A$ lie on the imaginary axis. If $r(A) \cap i R$ is countable, then $(\mathrm{T}(\mathrm{t}))_{\mathrm{t} \geq 0}$ is stable.

Our main result is the following theorem

Theorem 4 The $\mathrm{C}_{0}$-semigroup $\mathrm{e}^{\mathrm{t} \mathcal{A}}$ is strongly stable in $\mathcal{H}$; i.e, for all $\mathrm{U}_{0} \in \mathcal{H}$, the solution of (17) satisfies

$$
\lim _{t \rightarrow \infty}\left\|e^{t \mathcal{A}} \mathrm{U}_{0}\right\|_{\mathcal{H}}=0
$$

For the proof of Theorem 4, we need the following two lemmas. 
Lemma $5 \mathcal{A}$ does not have eigenvalues on $i \mathbb{R}$.

\section{Proof.}

Case 1: We will argue by contraction. Let us suppose that there $\tilde{\lambda} \in \mathbb{R}, \tilde{\lambda} \neq 0$ and $\mathrm{U} \neq 0$, such that

$$
\mathcal{A U}=i \tilde{\lambda} \mathrm{U}
$$

Then, we get

$$
\left\{\begin{array}{l}
i \tilde{\lambda} u-v=0 \\
i \tilde{\lambda} v-\mu \Delta u-(\mu+\lambda) \nabla(\operatorname{div} u)+\zeta \int_{-\infty}^{+\infty} \omega(\xi) \psi(x, \xi) d \xi+a_{2} v=0, \\
i \tilde{\lambda} \psi+\left(\xi^{2}+\kappa\right) \psi-z(x, 1) \omega(\xi)=0 \\
i \tilde{\lambda} z(x, \rho)+\tau^{-1} z_{\rho}(x, \rho)=0 .
\end{array}\right.
$$

Then, from (20) we have

$$
v=0, \quad z(x, 1)=0
$$

Hence, from $(35)_{3}$ and (36) we obtain

$$
u \equiv 0, \quad \psi \equiv 0
$$

Note that $(35)_{4}$ gives us $z=v e^{-i \tilde{\lambda} \rho \tau}=0$ as the unique solution of $(35)_{4}$. Hence $\mathrm{U} \equiv 0$.

Now if $\tilde{\lambda}=0$, inserting $(35)_{1}$ into $(35)_{2}$, we deduce that

$$
\{-\mu \Delta u-(\mu+\lambda) \nabla(\operatorname{div} u)=0, u=0 \text { in } \Gamma .
$$

Multiplying by $\bar{u}$, integrating over $\Omega$ we have

$$
\|\nabla u\|_{\mathrm{L}^{2}(\Omega)}^{2}+\|\operatorname{div} u\|_{\mathrm{L}^{2}(\Omega)}^{2}=0 .
$$

Hence $\mathfrak{u}=0$. Then $\mathrm{U} \equiv 0$.

Lemma 6 We have

$$
i \mathbb{R} \subset \rho(\mathcal{A}) .
$$


Proof. We will prove that the operator $i \tilde{\lambda} I-\mathcal{A}$ is surjective for $\tilde{\lambda} \neq 0$. For this purpose, let $\mathrm{G}=\left(\mathrm{G}_{1}, \mathrm{G}_{2}, \mathrm{G}_{3}, \mathrm{G}_{4}\right)^{\top} \in \mathcal{H}$, we seek $\mathrm{X}=(\mathrm{u}, \nu, \psi, z)^{\top} \in \mathrm{D}(\mathcal{A})$ of solution of the following equation

$$
(i \tilde{\lambda} \mathrm{I}-\mathcal{A}) \mathrm{X}=\mathrm{G} .
$$

Equivalently, we have

$$
\left\{\begin{array}{l}
i \tilde{\lambda} u-v=G_{1} \\
i \tilde{\lambda} v-\mu \Delta u-(\mu+\lambda) \nabla(\operatorname{div} u)+\zeta \int_{-\infty}^{+\infty} \omega(\xi) \psi(x, \xi) d \xi+a_{2} v=G_{2}, \\
i \tilde{\lambda} \psi+\left(\xi^{2}+\kappa\right) \psi-z(x, 1) \omega(\xi)=G_{3} \\
i \tilde{\lambda} z(x, \rho)+\tau^{-1} z_{\rho}(x, \rho)=G_{4}
\end{array}\right.
$$

From $(41)_{1}$ and $(41)_{2}$, we have

$-\tilde{\lambda}^{2} u-\mu \Delta u-(\mu+\lambda) \nabla(\operatorname{div} u)+\zeta \int_{-\infty}^{+\infty} \omega(\xi) \psi(x, \xi) d \xi+a_{2} v(t)=\left(G_{2}+i \tilde{\lambda} G_{1}\right)$

with $\mathfrak{u}_{\mid \Gamma}=0$. Solving system (42) is equivalent to finding $u \in\left(H^{2} \cap H_{0}^{1}(\Omega)\right)^{n}$ such that

$$
\left\{\begin{array}{l}
\sum_{j=1}^{n} \int_{\Omega}\left(-\left(\tilde{\lambda}^{2}+i \tilde{\lambda} a_{2}\right) u_{j} \bar{w}_{j}+\mu \nabla u_{j} \nabla \bar{w}_{j} d x\right)+(\mu+\lambda) \int_{\Omega}(\operatorname{div} u)(\operatorname{div} \bar{w}) d x \\
+i \tilde{\lambda} \theta \sum_{j=1}^{n} \int_{\Omega} u_{j} \bar{w}_{j} e^{-\tilde{\lambda} \tau} d x=\sum_{j=1}^{n} \int_{\Omega}\left(g_{2}^{j}(x)+\left(i \tilde{\lambda}+a_{2}\right) g_{1}^{j}(x)\right) \bar{w}_{j} d x \\
-\zeta \sum_{j=1}^{n} \int_{\Omega} \bar{w}_{j}\left(\int_{-\infty}^{\infty} \frac{\omega(\xi) g_{3}^{j}(x, \xi)}{\xi^{2}+k+i \tilde{\lambda}} d \xi\right) d x-\theta \sum_{j=1}^{n} \int_{\Omega} \bar{w}_{j} z_{0}(x) d x
\end{array}\right.
$$

for all $w \in\left(H_{0}^{1}(\Omega)\right)^{n}$. We can rewrite $(43)$ as

$$
-\left(\mathrm{L}_{\tilde{\lambda}} \mathrm{u}, \boldsymbol{w}\right)_{\left(\left(\mathrm{H}_{0}^{1}(\Omega)\right)^{n},\left(\left(\mathrm{H}_{0}^{1}(\Omega)\right)^{\prime}\right)^{n}\right)}+\mathrm{a}_{\left(\mathrm{H}_{0}^{1}(\Omega)\right)^{n}}(\mathrm{u}, \boldsymbol{w})=l(w)
$$

with the sesquilinear form defined by

$$
\begin{aligned}
a_{\left(H_{0}^{1}(\Omega)\right)^{n}}(u, w) & =\mu \sum_{j=1}^{n} \int_{\Omega} \nabla u_{j} \nabla \bar{w}_{j} d x+i \tilde{\lambda} a_{2} \sum_{j=1}^{n} \int_{\Omega} u_{j} \bar{w}_{j} d x \\
& +i \tilde{\lambda} \theta \sum_{j=1}^{n} \int_{\Omega} u_{j} \bar{w}_{j} e^{-\tilde{\lambda} \tau} d x
\end{aligned}
$$


and

$$
\left(\mathrm{L}_{\tilde{\lambda}} \mathrm{u}, \boldsymbol{w}\right)_{\left(\left(\mathrm{H}_{0}^{1}(\Omega)\right)^{n},\left(\left(\mathrm{H}_{0}^{1}(\Omega)\right)^{\prime}\right)^{\mathrm{n}}\right)}=\sum_{j=1}^{n} \int_{\Omega} \tilde{\lambda}^{2} u_{j} \bar{w}_{j} d x .
$$

Using the compactness of the embedding from $\mathrm{L}^{2}(\Omega)$ into $\mathrm{H}^{-1}(\Omega)$ and from $H_{0}^{1}(\Omega)$ into $L^{2}(\Omega)$ we deduce that the operator $L_{\tilde{\lambda}}$ is compact from $\left(L^{2}(\Omega)\right)^{n}$ into $\left(\mathrm{L}^{2}(\Omega)\right)^{\mathrm{n}}$. Consequently, by the Fredholm alternative, proving the existence of a solution $\mathfrak{u}$ of (44) reduces to proving that there is not a nontrivial solution for $(44)$ for $l \equiv 0$. Indeed if there exists $u \neq 0$, such that

$$
\left(\mathrm{L}_{\lambda} \mathrm{u}, \boldsymbol{w}\right)_{\left(\left(\mathrm{H}_{0}^{1}(\Omega)\right)^{n},\left(\left(\mathrm{H}_{0}^{1}(\Omega)\right)^{\prime}\right)^{n}\right)}=\mathrm{a}_{\left(\mathrm{H}_{0}^{1}(\Omega)\right)^{n}}(\mathrm{u}, w) \quad \forall w \in\left(\mathrm{H}_{0}^{1}(\Omega)\right)^{n},
$$

then $i \tilde{\lambda}$ is an eigenvalue of $\mathcal{A}$. Therefore from Lemma 5 we deduce that $u=0$.

Now, if $\tilde{\lambda}=0$, the system (41) is reduced to the following system

$$
\left\{\begin{array}{l}
v=-G_{1} \\
-\mu \Delta u-(\mu+\lambda) \nabla(\operatorname{div} u)+\zeta \int_{-\infty}^{+\infty} \omega(\xi) \psi(x, \xi) d \xi+a_{2} v=G_{2} \\
\left(\xi^{2}+\kappa\right) \psi-z(x, 1) \omega(\xi)=G_{3} \\
\tau^{-1} z_{\rho}(x, \rho)=G_{4} .
\end{array}\right.
$$

Solving system (46) is equivalent to finding $u \in\left(H^{2} \cap H_{0}^{1}(\Omega)\right)^{n}$ such that

$$
\begin{aligned}
& \mu \sum_{j=1}^{n} \int_{\Omega} \nabla u_{j} \nabla \bar{w}_{j} d x+(\mu+\lambda) \int_{\Omega}(\operatorname{div} u)(\operatorname{div} \bar{w}) d x=\sum_{j=1}^{n} \int_{\Omega} g_{2}^{j} \bar{w}_{j} d x \\
& +\left(\zeta \int_{-\infty}^{\infty} \frac{\omega^{2}(\xi)}{\xi^{2}+\kappa} d \xi+a_{2}\right) \sum_{j=1}^{n} \int_{\Omega} g_{1}^{j} \bar{w}_{j} d x \\
& -\tau \zeta \int_{-\infty}^{\infty} \frac{\omega^{2}(\xi)}{\xi^{2}+k} d \xi \sum_{j=1}^{n} \int_{\Omega} \int_{0}^{1} g_{4}^{j}(x, s) d s \bar{w}_{j} d x \\
& -\zeta \sum_{j=1}^{n} \int_{\Omega} \bar{w}_{j} \int_{-\infty}^{\infty} \frac{\omega(\xi) g_{3}^{j}(x, \xi)}{\xi^{2}+k} d \xi d x .
\end{aligned}
$$

for all $w \in\left(H_{0}^{1}(\Omega)\right)^{n}$. Consequently, problem (47) is equivalent to the problem

$$
\mathcal{B}(u, w)=\mathcal{L}(w)
$$

where the sesquilinear form $\mathcal{B}:\left(H_{0}^{1}(\Omega)\right)^{n} \times\left(H_{0}^{1}(\Omega)\right)^{n} \rightarrow \mathbb{C}$ and the antilinear form $\mathcal{L}:\left(\mathrm{H}_{0}^{1}(\Omega)\right)^{\mathrm{n}} \rightarrow \mathbb{C}$ are defined by

$$
\mathcal{B}(u, w)=\mu \sum_{j=1}^{n} \int_{\Omega} \nabla u_{j} \nabla \bar{w}_{j} d x+(\mu+\lambda) \int_{\Omega}(\operatorname{div} u)(\operatorname{div} \bar{w}) d x
$$


and

$$
\begin{aligned}
\mathcal{L}(w) & =\sum_{j=1}^{n} \int_{\Omega} g_{2}^{j} \bar{w}_{j} d x+\left(\zeta \int_{-\infty}^{\infty} \frac{\omega^{2}(\xi)}{\xi^{2}+\kappa} d \xi+a_{2}\right) \sum_{j=1}^{n} \int_{\Omega} g_{1}^{j} \bar{w}_{j} d x \\
& -\tau \zeta \int_{-\infty}^{\infty} \frac{\omega^{2}(\xi)}{\xi^{2}+\kappa} d \xi \sum_{j=1}^{n} \int_{\Omega} \int_{0}^{1} g_{4}^{j}(x, s) d s \bar{w}_{j} d x \\
& -\zeta \sum_{j=1}^{n} \int_{\Omega} \bar{w}_{j} \int_{-\infty}^{\infty} \frac{\omega(\xi) g_{3}^{j}(x, \xi)}{\xi^{2}+\kappa} d \xi d x .
\end{aligned}
$$

It is easy to verify that $\mathcal{B}$ is continuous and coercive, and $\mathcal{L}$ is continuous. So applying the Lax-Milgram theorem, we deduce that for all $w \in\left(H_{0}^{1}(\Omega)\right)^{n}$ problem (48) admits a unique solution $u \in\left(H_{0}^{1}(\Omega)\right)^{n}$. Applying the classical elliptic regularity, it follows from (47) that $u \in\left(H^{2}(\Omega)\right)^{n}$. Therefore, the operator $\mathcal{A}$ is surjective.

\section{$5 \quad$ Exponential stability}

The necessary and suficient conditions for the exponential stability of the $\mathrm{C}_{0^{-}}$ semigroup of contractions on a Hilbert space were obtained by Gearhart [7] and Huang [9] independently, see also Pruss [15]. We will use the following result due to Gearhart.

Theorem 5 ([15]- [9]) Let $\mathrm{S}(\mathrm{t})=\mathrm{e}^{\mathcal{A} \mathrm{t}}$ be a $\mathrm{C}_{0}$-semigroup of contractions on Hilbert space $\mathcal{H}$. Then $\mathbf{S}(\mathrm{t})$ is exponentially stable if and only if

$$
\rho(\mathcal{A}) \supseteq\{i \beta: \beta \in \mathbb{R}\} \equiv i \mathbb{R}
$$

and

$$
\overline{\lim }_{|\beta| \rightarrow \infty}\left\|(i \beta I-\mathcal{A})^{-1}\right\|_{\mathcal{L}(\mathcal{H})}<\infty .
$$

Our main result is as follows.

Theorem 6 The semigroup $\mathrm{S}_{\mathcal{A}}(\mathrm{t})_{\mathrm{t} \geq 0}$ generated by $\mathcal{A}$ is exponentially stable.

Proof. We will need to study the resolvent equation $(i \tilde{\lambda}-\mathcal{A}) \mathrm{U}=\mathrm{G}$, for $\lambda \in \mathbb{R}$, 
namely

$$
\left\{\begin{array}{l}
i \tilde{\lambda} u-v=G_{1} \\
i \tilde{\lambda} v-\mu \Delta u-(\mu+\lambda) \nabla(\operatorname{div} u)+\zeta \int_{-\infty}^{+\infty} \omega(\xi) \psi(x, \xi) d \xi+a_{2} v=G_{2}, \\
i \tilde{\lambda} \psi+\left(\xi^{2}+\kappa\right) \psi-z(x, 1) \omega(\xi)=G_{3} \\
i \tilde{\lambda} z(x, \rho)+\tau^{-1} z_{\rho}(x, \rho)=G_{4}
\end{array}\right.
$$

where $G=\left(G_{1}, G_{2}, G_{3}, G_{4}\right)^{\top}$. Taking inner product in $\mathcal{H}$ with $\mathrm{U}$ and using (20) we get

$$
|\operatorname{Re}\langle\mathcal{A U}, \mathrm{U}\rangle| \leq\|\mathrm{U}\|_{\mathcal{H}}\|\mathrm{G}\|_{\mathcal{H}} .
$$

This implies that

$$
\sum_{j=1}^{n} \int_{\Omega} v_{j}^{2}(x) d x, \quad \sum_{j=1}^{n} \int_{\Omega} z_{j}^{2}(x, 1) d x \leq C\|\mathrm{U}\|_{\mathcal{H}}\|\mathrm{G}\|_{\mathcal{H}} .
$$

From $(53)_{3}$, we obtain

$$
\psi=\frac{z(x, 1) \omega(\xi)+G_{3}}{i \tilde{\lambda}+\xi^{2}+\kappa} .
$$

Then

$$
\begin{aligned}
\|\psi\|_{\mathrm{L}^{2}(\Omega \times(-\infty,+\infty))} \leq & \left\|\frac{\omega(\xi)}{\tilde{i} \tilde{\lambda}+\xi^{2}+\kappa}\right\|_{\mathrm{L}^{2}(-\infty,+\infty)}\|z(x, 1)\|_{\mathrm{L}^{2}(\Omega)} \\
& +\left\|\frac{\mathrm{G}_{3}}{\tilde{i} \tilde{\lambda}+\xi^{2}+\kappa}\right\|_{\mathrm{L}^{2}(\Omega \times(-\infty,+\infty))} \\
\leq & \left(2(1-\sigma) \frac{\pi}{\sin \sigma \pi}(|\tilde{\lambda}|+\kappa)^{\sigma-2}\right)^{\frac{1}{2}}\|z(x, 1)\|_{\mathrm{L}^{2}(\Omega)} \\
& +\frac{\sqrt{2}}{|\tilde{\lambda}|+\kappa}\left\|\mathrm{G}_{3}\right\|_{\mathrm{L}^{2}(\Omega \times(-\infty,+\infty))} .
\end{aligned}
$$

Similarly, we have

$$
\begin{aligned}
\|\xi \psi\|_{\mathrm{L}^{2}(\Omega \times(-\infty,+\infty)) \leq} & \left\|\frac{\xi \omega(\xi)}{i \tilde{\lambda}+\xi^{2}+\kappa}\right\|_{\mathrm{L}^{2}(-\infty,+\infty)}\|z(x, 1)\|_{\mathrm{L}^{2}(\Omega)} \\
& +\left\|\frac{\xi \mathrm{G}_{3}}{i \tilde{\lambda}+\xi^{2}+\kappa}\right\|_{\mathrm{L}^{2}(\Omega \times(-\infty,+\infty))} \\
\leq & \left(2 \sigma \frac{\pi}{\sin \sigma \pi}(|\tilde{\lambda}|+\kappa)^{\sigma-1}\right)^{\frac{1}{2}}\|z(x, 1)\|_{\mathrm{L}^{2}(\Omega)} \\
& +\frac{\sqrt{2}}{\sqrt{|\tilde{\lambda}|+\kappa}}\left\|\mathrm{G}_{3}\right\|_{\mathrm{L}^{2}(\Omega \times(-\infty,+\infty))}
\end{aligned}
$$


Let us introduce the following notation

$$
\mathcal{I}_{\mathfrak{u}}(x)=\sum_{j=1}^{n}\left(\left|v_{j}(x)\right|^{2}+\mu\left|\nabla u_{j}(x)\right|^{2}\right)+(\mu+\lambda)|\operatorname{div} u(x)|^{2}
$$

and

$$
\mathcal{E}_{\mathfrak{u}}=\int_{\Omega} \mathcal{I}_{\mathfrak{u}}(x) \mathrm{d} x
$$

Lemma 7 We have that

$$
\mathcal{E}_{\mathrm{u}} \leq \mathrm{c}\|\mathrm{G}\|_{\mathcal{H}}^{2}+\mathrm{c}^{\prime}\|\mathrm{G}\|_{\mathcal{H}}\|\mathrm{U}\|_{\mathcal{H}} .
$$

for positive constants $\mathrm{c}$ and $\mathrm{c}^{\prime}$.

Proof. Multiplying the equation $(53)_{2}$ by $\bar{u}$, integrating on $\Omega$ we obtain

$$
\begin{aligned}
& -\int_{\Omega} v_{j}\left(\overline{\hat{i} \tilde{\lambda} u_{j}}\right) d x+\mu \int_{\Omega}\left|\nabla u_{j}\right|^{2} d x+(\mu+\lambda) \int_{\Omega}(\operatorname{div} u) \frac{\partial \overline{u_{j}}}{\partial x_{j}} d x \\
& +\zeta \int_{\Omega} \bar{u}_{j}\left(\int_{-\infty}^{+\infty} \omega(\xi) \psi_{j}(x, \xi) d \xi\right) d x+a_{2} \int_{\Omega} \bar{u}_{j} v_{j} d x=\int_{\Omega} \bar{u} g_{2}^{j} d x .
\end{aligned}
$$

From $(53)_{1}$, we have $i \tilde{\lambda} u_{j}=v_{j}+g_{1}^{j}$. Then

$$
\begin{aligned}
& -\int_{\Omega}\left|v_{j}\right|^{2} \mathrm{~d} x+\mu \int_{\Omega}\left|\nabla u_{j}\right|^{2} \mathrm{~d} x+(\mu+\lambda) \int_{\Omega}(\operatorname{div} u) \frac{\partial \bar{u}_{j}}{\partial x_{j}} d x \\
& +\zeta \int_{\Omega} \bar{u}_{j}\left(\int_{-\infty}^{+\infty} \omega(\xi) \psi_{j}(x, \xi) d \xi\right) d x+a_{2} \int_{\Omega} \bar{u}_{j} v_{j} d x \\
& =\int_{\Omega} \overline{u_{j}} g_{2}^{j} d x+\int_{\Omega} v_{j} \bar{g}_{1}^{j} d x .
\end{aligned}
$$

Hence

$$
\begin{aligned}
& -\sum_{j=1}^{n} \int_{\Omega}\left|v_{j}\right|^{2} d x+\mu \sum_{j=1}^{n} \int_{\Omega}\left|\nabla u_{j}\right|^{2} d x+(\mu+\lambda) \int_{\Omega}|\operatorname{div} u|^{2} d x \\
& +\zeta \sum_{j=1}^{n} \int_{\Omega} \bar{u}_{j}\left(\int_{-\infty}^{+\infty} \omega(\xi) \psi_{j}(x, \xi) d \xi\right) d x+a_{2} \sum_{j=1}^{n} \int_{\Omega} \bar{u}_{j} v_{j} d x \\
& =\sum_{j=1}^{n} \int_{\Omega} \bar{u}_{j} g_{2}^{j} d x+\sum_{j=1}^{n} \int_{\Omega} v_{j} \bar{g}_{1}^{\bar{j}} d x .
\end{aligned}
$$


We can estimate

$$
\begin{aligned}
& \left|\int_{\Omega} \bar{u}_{j}\left(\int_{-\infty}^{+\infty} \omega(\xi) \psi_{j}(x, \xi) d \xi\right) d x\right| \\
& \leq\left\|u_{j}\right\|_{L^{2}(\Omega)}\left(\int_{-\infty}^{+\infty} \frac{\omega^{2}(\xi)}{\xi^{2}+\kappa} d \xi\right)^{\frac{1}{2}}\left(\int_{\Omega} \int_{-\infty}^{+\infty}\left(\xi^{2}+\kappa\right)\left|\psi_{j}(x, \xi)\right|^{2} d \xi d x\right)^{\frac{1}{2}} \\
& \leq \frac{\varepsilon}{2}\left(\int_{-\infty}^{+\infty} \frac{\omega^{2}(\xi)}{\xi^{2}+\kappa} d \xi\right)\left\|u_{j}\right\|_{L^{2}(\Omega)}^{2}+\frac{1}{2 \varepsilon} \int_{\Omega} \int_{-\infty}^{+\infty}\left(\xi^{2}+\kappa\right)\left|\psi_{j}(x, \xi)\right|^{2} d \xi d x \\
& \leq \frac{\varepsilon}{2} \mathrm{C}(\Omega)\left(\int_{-\infty}^{+\infty} \frac{\omega^{2}(\xi)}{\xi^{2}+\kappa} d \xi\right)\left\|\nabla u_{j}\right\|_{L^{2}(\Omega)}^{2}+\frac{1}{2 \varepsilon} \int_{\Omega} \int_{-\infty}^{+\infty}\left(\xi^{2}+\kappa\right)\left|\psi_{j}(x, \xi)\right|^{2} d \xi d x, \\
& \left|\int_{\Omega} \overline{u_{j}} v_{j} d x\right| \leq\left\|u_{j}\right\|_{L^{2}(\Omega)}\left\|v_{j}\right\|_{L^{2}(\Omega)} \leq \frac{\varepsilon}{2} C(\Omega)\left\|\nabla u_{j}\right\|_{L^{2}(\Omega)}^{2}+\frac{1}{2 \varepsilon}\left\|v_{j}\right\|_{L^{2}(\Omega)}^{2}, \\
& \left|\int_{\Omega} \overline{u_{j}} g_{2}^{j} d x\right| \leq \frac{\varepsilon}{2} C(\Omega)\left\|\nabla u_{j}\right\|_{L^{2}(\Omega)}^{2}+\frac{1}{2 \varepsilon}\left\|g_{2}^{j}\right\|_{L^{2}(\Omega)}^{2}, \\
& \left|\int_{\Omega} v_{j} g_{1}^{j} d x\right| \leq \frac{\varepsilon}{2}\left\|v_{j}\right\|_{L^{2}(\Omega)}^{2}+\frac{1}{2 \varepsilon}\left\|g_{1}^{j}\right\|_{L^{2}(\Omega)}^{2} .
\end{aligned}
$$

Choosing $\varepsilon$ small enough, we conclude (59).

Moreover, the equation $(53)_{4}$ has a unique solution

$$
\begin{aligned}
z(x, \rho) & =e^{-i \tau \tilde{\lambda} \rho} z(x, 0)+\tau e^{-i \tau \tilde{\lambda} \rho} \int_{0}^{\rho} e^{-i \tau \tilde{\lambda} r} G_{4}(x, r) \\
& =e^{-i \tau \tilde{\lambda} \rho} v(x)+\tau e^{-i \tau \tilde{\lambda} \rho} \int_{0}^{\rho} e^{-i \tau \tilde{\lambda} r} G_{4}(x, r) d r .
\end{aligned}
$$

Then

$$
\|z(x, \rho)\|_{\mathrm{L}^{2}(\Omega \times(0,1))} \leq\|v(x)\|_{\mathrm{L}^{2}(\Omega)}+\tau\left\|\mathrm{G}_{4}(x, \rho)\right\|_{\mathrm{L}^{2}(\Omega \times(0,1))} .
$$

Finally, (57), (59) and (63) imply that

$$
\|\mathrm{U}\|_{\mathcal{H}} \leq \mathrm{C}\|\mathrm{G}\|_{\mathcal{H}}
$$

for a positive constant $\mathrm{C}$. The conclusion then follows by applying Theorem 5 .

Remark 2 We can extend the results of this paper to more general measure density instead of (1), that is $\omega$ is an even nonnegative measurable function such that

$$
\int_{-\infty}^{\infty} \frac{\omega(\xi)^{2}}{1+\xi^{2}} d \xi<\infty
$$




\section{References}

[1] C. Abdallah, P. Dorato, J. Benitez-Read \& R. Byrne, Delayed positive feedback can stabilize oscillatory system, ACC, San Francisco, (1993), 3106-3107.

[2] W. Arendt \& C. J. K. Batty, Tauberian theorems and stability of oneparameter semigroups, Trans. Am. Math. Soc., 306 (1988), 837-852.

[3] R. L. Bagley \& P. J. Torvik, A theoretical basis for the application of fractional calculus to viscoelasticity, J. Rheology., 27 (1983), 201-210.

[4] R. L. Bagley \& P. J. Torvik, On the appearance of the fractional derivative in the behavior of real material, J. Appl. Mech., 51 (1983), 294-298.

[5] A. Benaissa, S. Gaouar, Asymptotic stability for the Lamé system with fractional boundary damping, Comput. Math. Appl., 77 (2019)-5, 13311346.

[6] H. Brézis, Operateurs Maximaux Monotones et semi-groupes de contractions dans les espaces de Hilbert, Notas de Matemàtica (50), Universidade Federal do Rio de Janeiro and University of Rochester, North-Holland, Amsterdam, (1973).

[7] L. Gearhart, Spectral theory for contraction semigroups on Hilbert spaces, Trans. Amer. Math. Soc. 236 (1978), 385-394.

[8] H. Haddar, J. R Li and D. Matignon, Efficient solution of a wave equation with fractional-order dissipative terms, J. Comput. Appl. Math., 234 (2010)-6, 2003-2010.

[9] F. Huang, Characteristic conditions for exponential stability of linear dynamical systems in Hilbert spaces, Ann. Differ. Equ., 1 (1985), 43-55.

[10] I. Lyubich Yu \& V. Q. Phóng, Asymptotic stability of linear differential equations in Banach spaces, Studia Mathematica, 88 (1988)-(1), 37-42.

[11] F. Mainardi \& E. Bonetti, The applications of real order derivatives in linear viscoelasticity, Rheol. Acta, 26 (1988), 64-67.

[12] B. Mbodje, Wave energy decay under fractional derivative controls, IMA Journal of Mathematical Control and Information., 23 (2006), 237-257. 
[13] S. Nicaise and C. Pignotti, Stability and instability results of the wave equation with a delay term in the boundary or internal feedbacks, SIAM J. Control Optim., 45 (2006)-5, 1561-1585.

[14] I. Podlubny, Fractional differential equations, Mathematics in Science and Engineering, 198 (1999), Academic Press.

[15] J. Prüss, On the spectrum of $\mathrm{C}_{0}$-semigroups, Transactions of the American Mathematical Society. 284 (1984)-2, 847-857.

[16] S. G. Samko, A. A. Kilbas, O. I. Marichev, emphFractional integrals and derivatives, Amsterdam: Gordon and Breach (1993) [Engl. Trans. from the Russian (1987)].

[17] I. H. Suh \& Z. Bien, Use of time delay action in the controller design, IEEE Trans. Autom. Control, 25 (1980) 600-603. 